\title{
Reduced Hepatic Bilirubin Uridine Diphosphate Glucuronyl Transferase and Uridine Diphosphate Glucose Dehydrogenase Activity in the Human Fetus
}

\author{
BERTRAM F. FELSHER, ${ }^{(22)}$ JACK E. MAIDMAN, NOEMI M. CARPIO, KENNETH VANCOUVERING, \\ AND MORTON M. WOOLLEY

\begin{abstract}
Long Beach Veterans Administration Hospital and University of California, Irvine, School of Medicine; Martin Luther King Hospital and Charles Drew Postgraduate Medical School; and Childrens Hospital and University of Southern California School of Medicine; Los Angeles, California
\end{abstract}

Summary

Hepatic bilirubin uridine diphosphate glucuronyl transferase (UDPG-T) activity was 0.14 and 0.22 units in two fetuses aged 17 and 22 weeks, respectively, and less than 0.1 unit in 15 fetuses, aged 8-19 weeks compared to 0.68-1.99 units in 21 normal adults.

Hepatic uridine diphosphate glucose dehydrogenase (UDPG-D) activity in 14 fetuses, aged $8-18$ weeks, ranged from 6.2-15.0 units $($ mean $=11.3 \pm 0.7)$ compared to $28.8-49.2$ units $($ mean $=39.6$ \pm 2.5 ) in eight normal adults $(P<0.001)$. There was no correlation between UDPG-D activity and gestational age. The hepatic UDPG-D activity was 16.5 units in a 33-day-old full term, female infant, $\mathbf{4 2 . 4}$ and $\mathbf{2 4 . 3}$ units in two 2-year-old infants, respectively, and 24.3 units in a 5.5-year-old child. In three human fetuses, the apparent $K_{\mathrm{m}}$ UDPG was $0.54 \times 10^{-4} \mathrm{M}$.

Thus, both hepatic bilirubin UDPG-T and UDPG-D activity are markedly reduced in the human fetus during the second trimester of gestation. Retarded development of hepatic UDPG-D may extend beyond the first month of life.

\section{Speculation}

The developmental pattern for hepatic bilirubin UDPG-T and UDPG-D in man are similar. Reduced capacity for uridine diphosphate glucuronic acid (UDPGA) formation may be an additional factor in the pathogenesis of physiologic jaundice of the newborn.

Conjugation of bilirubin is a virtual requirement for the excretion of bilirubin from liver to bile in normal man (1). The predominant conjugating moiety for bilirubin in human bile is glucuronic acid, although the structure of the conjugates may be more complex than a simple glucuronide $(10,11,14)$. The glucuronidation of bilirubin involves two reactions. The initial reaction, monoglucuronidation $(9,12)$ occurs in the hepatic endoplasmic reticulum where a specific enzyme, bilirubin UDPG-T catalyzes the transfer of glucuronic acid from a specific glucuronyl donor, UDPGA (6). UDPGA is formed in the liver cytosol from the enzymatic oxidation of UDP-glucose by UDPG-D; NAD serves as the hydrogen ion acceptor (18). An enzyme located in the liver plasma membrane catalyzes the transglucuronidation of bilirubin monoglucuronide resulting in formation of bilirubin diglucuronide and unconjugated bilirubin (13). The overall rate of hepatic bilirubin glucuronidation is controlled by the availability of UDPGA (7) as well as the activity of UDPG-T.
Information concerning the hepatic capacity for bilirubin glucuronidation and UDPGA formation in the human fetus is not available. The finding that the hepatic UDPGA concentration in two normal 3- to 4-month-old human fetuses was $10 \%$ of that in fresh adult mouse liver (5) is difficult to interpret because of the lack of comparable data in adult human liver and the marked lability of UDPGA in devitalized tissue (6). The earlier observation that hepatic UDPG-D activity is reduced in fetal and newborn guinea pig liver (3) suggested the possibility that a similar phenomenon might occur in man. Data on the activity of hepatic bilirubin UDPG-T and UDPG-D in the human fetus are not available. In the present study, hepatic bilirubin UDPG-T and UDPG-D activities were assayed in human fetuses aborted by hysterectomy.

\section{MATERIALS AND METHODS}

The livers of 17 dead fetuses were obtained from cases of elective abortion by hysterectomy performed for gynecologic reasons. Immediately after hysterectomy, the uterus was placed in ice, and within $15 \mathrm{~min}$, the fetus was removed, weighed, and measured, and the liver was removed and quick frozen in liquid nitrogen. Only intact, normal-appearing fetuses were eligible for the study. Gestational age of the fetus was determined by crownrump measurement according to the standards of Streeter (17). In addition, portions of liver biopsies taken for histologic examinations in adults and children were used to determine normal enzymic activity. Informed consent was obtained.

Homogenates were prepared by grinding $10-20 \mathrm{mg}$ liver in 10 vol homogenizing medium in an ice bath. The mixture was homogenized in a motor-driven, ground-glass homogenizer at a constant speed of $400 \mathrm{rpm}$ for $1 \mathrm{~min}$.

Hepatic bilirubin UDPG-T activity was assayed by a micromodification (2) of the method of Van Roy and Heirwegh (19) which employs bilirubin as the aglycone and digitonin as an enzyme activator. Hepatic UDPG-D activity of homogenates prepared in 10 vol $0.15 \mathrm{M} \mathrm{KCl}$ was assayed according to a modification of the radiochemical method described by Davies and Dickinson (4). The standard assay mixture $(0.06 \mathrm{ml})$ contained $100 \mathrm{mM}$ glycine/ $\mathrm{NaOH}$ buffer, $\mathrm{pH} 8.7,3 \mathrm{mM}$ NAD, $0.75 \mathrm{mM}$ UDP-[U. $\left.{ }^{14} \mathrm{C}\right]$ glucose $\left(4.8 \times 10^{5} \mathrm{cpm}, 12.03 \mathrm{mCi} / \mathrm{mmol}\right)$, and 0.01 $\mathrm{ml}$ liver homogenate. The control mixture was the same except NAD was omitted. The reaction mixtures were incubated at $30^{\circ}$ for $10 \mathrm{~min}$. The nucleotides were hydrolyzed enzymatically and resultant sugars, glucose, and glucuronic acid, were separated on DEAE-cellulose-coated plastic sheets using ethanol-water $(1: 1)$ as the solvent. Glucuronic acid remains close to the origin and 
glucose moves near the solvent front. The radioactivity at these two sites was quantified in a liquid scintillation counter.

Liver protein concentration was measured according to the method of Lowry et al (16) using bovine albumin as standard. All assays were done in duplicate and the intraassay coefficients of variation were $5 \%$ or less. The Student's $t$-test for unpaired data was used for statistical analyses.

\section{RESULTS}

Hepatic UDPG-T activity (unit = milligrams of bilirubin conjugated per $g$ liver per $h$ ) was undetectable or markedly reduced in all fetuses. The UDPG-T activity in two fetuses, aged 17 and 22 weeks, was 0.14 and 0.22 units, respectively, and in 15 fetuses, aged 8-19 weeks, was less than 0.1 units (Table 1 ). The range of hepatic UDPG-T activity in 21 normal adults was $0.68-1.99$ units. The hepatic UDPG-T specific activity (micrograms of bilirubin conjugated per $\mathrm{mg}$ protein per $\mathrm{h}$ ) ranged from $0.1-1.2$ (mean \pm SEM $=0.58 \pm 0.17$ ) in the six fetuses, aged 15-22 weeks, with detectable enzymic activity compared to $3.2-16.5(9.2 \pm 0.7)$ in 14 normal adults.

Hepatic UDPG-D activity (unit = micromoles of UDPGA formed per $100 \mathrm{~g}$ liver per min) in 14 fetuses, aged 8-18 weeks, ranged from 6.2-15.0 units $(11.3 \pm 0.7)$ compared to $28.8-49.2$ units $(39.6 \pm 2.5)$ in eight normal adults $(P<0.001)$. The hepatic UDPG-D specific activity (nanomoles of UDPGA formed per $\mathrm{mg}$ protein per $\mathrm{min}$ ) ranged from $0.44-1.70(0.95 \pm 0.09)$ in the fetuses compared to 1.73-3.02 (2.64 \pm 0.17$)$ in seven normal adults $(P<$ $0.001)$. UDPG-D activity for these 10- to 18-week-old human fetuses was not correlated with gestational age $(r=0.34, P>0.2)$. In a 33-day-old, full term female infant who underwent hepatic

Table 1. UDPG-D and bilirubin UDPG-T activities of human fetal liver

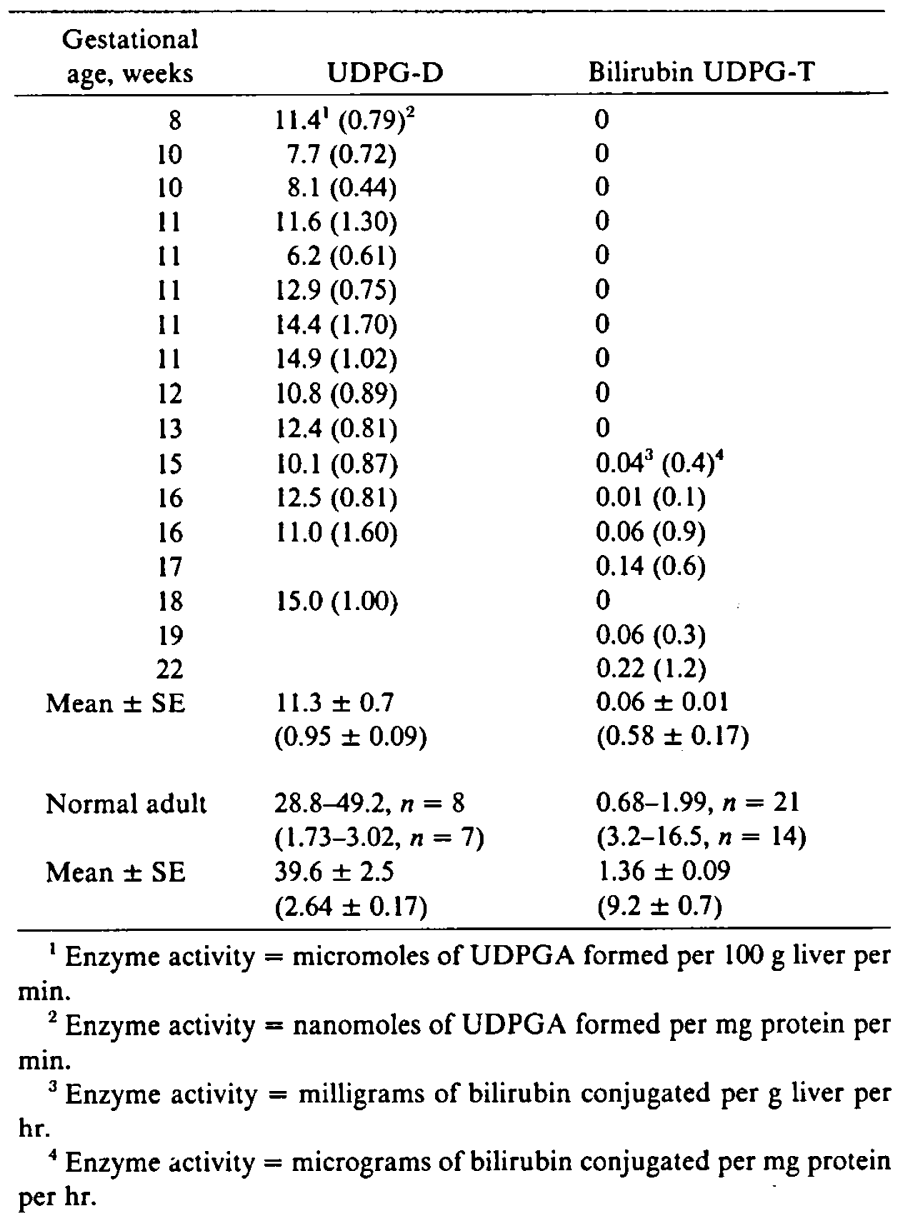

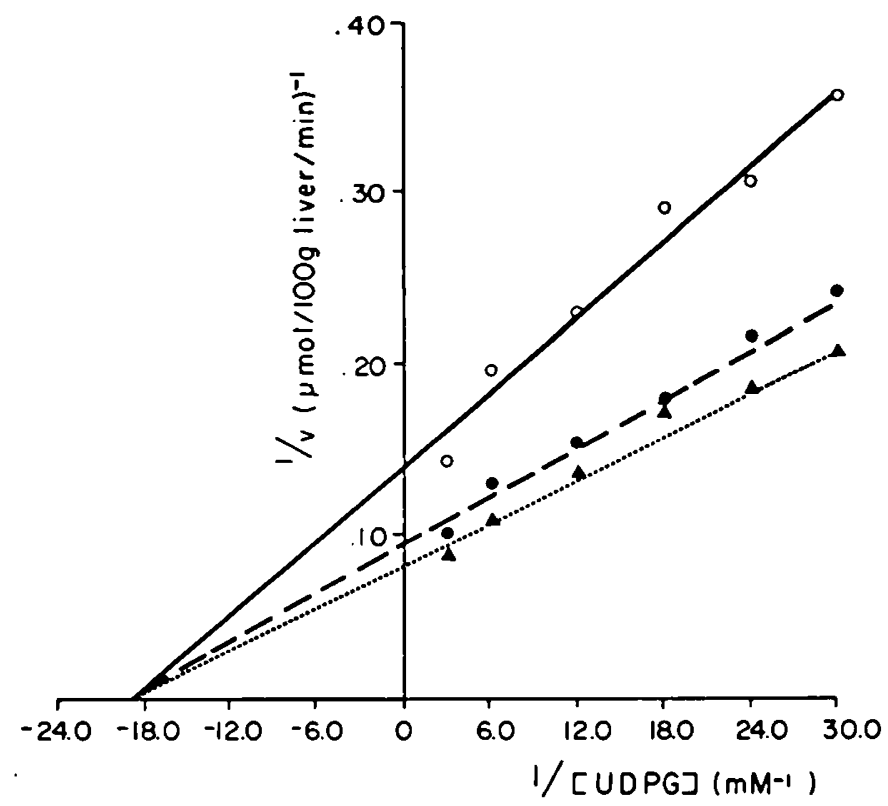

Fig. 1. Lineweaver-Burk plots obtained from UDPG-D in liver homogenates of human fetuses. $\mathrm{O}-\mathrm{O}$ : 11-week-old; old; $\Delta$-..- $\Delta$ : 8-week-old. Note the identical apparent $K_{m}$ values of enzyme from all sources.

surgery for a benign neoplasm, UDPG-D activity was 16.5 units, which is higher than fetal, but still somewhat lower than normal adult values. The enzymic activity in a normal 2 year old was 42.4 units. In another 2 year old it was 24.3 units and in a 5.5-year-old child it was 20.6 units.

The apparent $K_{m}$ UDPG was determined in liver homogenates from three human fetuses ( $8-11$ weeks old) by constructing Lineweaver-Burk plots (Fig. 1). The intercepts on the abscissa $\left(1 / \mathrm{K}_{\mathrm{m}}\right)$ were identical and the calculated $\mathrm{K}_{\mathrm{m}}$ value was $0.54 \times 10^{-4} \mathrm{M}$. Thus, the concentration of UDPG used in the UDPG-D assay $\left(7.5 \times 10^{-4} \mathrm{M}\right)$ is 14 -fold higher than the apparent $\mathrm{K}_{\mathrm{m}}$ UDPG for fetal hepatic UDPG-D. The apparent $K_{m}$ UDPG determined in homogenate of normal liver obtained surgically from a 60 -yearold man was $0.37 \times 10^{-4} \mathrm{M}$. Increasing the concentration of UDPGA 2-4 times that used in the standard UDPG-T incubation mixture was not associated with any increase in human fetal hepatic UDPG-T activity.

\section{DISCUSSION}

Information concerning the developmental pattern of hepatic bilirubin glucuronidation in man is scarce. In 1958, Lathe and Walker (15) reported the virtual absence of bilirubin UDPG-T activity in the livers of three premature infants (25-32 weeks of gestation) who expired $5 \mathrm{~min}$ to $98 \mathrm{hr}$ after birth. In a recent study of living human newborns with congenital gastrointestinal obstruction, the hepatic bilirubin UDPG-T activity was markedly reduced in 10 subjects ( $2-42$ days old) with unconjugated hyperbilirubinemia compared to 7 anicteric subjects (8). In these anicteric infants (19-71 days old), the hepatic bilirubin UDPG-T activities were within the low "normal" range for adults.

This study shows that the hepatic activities of both bilirubin UDPG-T, and UDPG-D when expressed in terms of liver weight, are markedly reduced in human fetuses aged 8-22 weeks compared to normal adults. When the enzymic activities were expressed in terms of liver protein, the differences between fetal and adult mean values were still highly significant, although in two fetuses, because of markedly reduced hepatic protein concentrations, the UDPG-D specific activities approached the normal adult range. An additional finding was markedly reduced hepatic 
UDPG-D activity in a 33-day-old normal full term human newborn. Kinetic studies in human fetal livers indicated that the concentrations of UDPG and UDPGA used in the UDPG-D and UDPG-T assays, respectively, were not rate limiting. The apparent $\mathrm{K}_{\mathrm{m}}$ UDPG in human fetal liver (8-11 weeks) was $0.54 \times 10^{-4} \mathrm{M}$. Loss of enzymic activity from delay in obtaining tissue after death was avoided by limiting the subjects to intact fetuses delivered by hysterectomy and quick freezing the liver promptly after delivery. Measurement of hepatic UDPGA concentration was not performed, however, because of the likelihood that the 15-min delay would lead to spuriously low values (unpublished observation).

Reduced in vitro hepatic UDPG-D activity suggests the possibility that in vivo UDPGA formation might be reduced. If, in fact, hepatic UDPGA concentration is reduced substantially below the $\mathrm{K}_{\mathrm{m}}$ UDPGA, then the rate of bilirubin glucuronidation might be significantly impaired. However, neither the $K_{m}$ UDPGA for bilirubin UDPG-T nor the actual concentration of UDPGA for human fetal liver are known.

The factors regulating the development of bilirubin UDPG-T and UDPG-D in fetal and newborn human liver are not known. It is unclear whether the reduced activity of these enzymes is a consequence of inactivation, diminished synthesis, or increased catabolism. In the present study, absence of demonstrable UDPG$T$ activity was observed even though the activator, digitonin, was added to the enzyme preparation. The small difference in the $\mathrm{K}_{\mathrm{m}}$ UDPG between fetal and adult liver tentatively suggests that altered affinity of enzyme for UDPG was not responsible for the reduced fetal hepatic UDPG-D activity. However, further determinations of $\mathrm{K}_{\mathrm{m}}$ UDPG in adult and fetal human liver are needed before a firm conclusion can be made regarding this point.

Studies in animals suggest that the development of fetal UDPG$T$ activity is under hormonal control. The administration of glucocorticoids to pregnant Wistar rats stimulated various fetal hepatic glucuronyl transferases, but not for bilirubin or other late developing transferases (20). The available evidence indicates that in man, the specific bilirubin glucuronyl transferase attains adult levels after birth. Thus, it would seem unlikely that glucocorticoids are the trigger for the developmental surge for bilirubin UDPG-T in man.

\section{REFERENCES AND NOTES}

I. Billing. B. H.: Bilirubin metabolism. In: L. Schiff: Diseases of the Liver, Ed. 4. p. 287 (J. B. Lippincott Co., Philadelphia. 1975).

2. Black. M.. Billing. B. H., and Heirwegh. K. P. M.: Determination of bilirubin
UDP-glucuronyl transferase activity in needle-biopsy specimens of human liver. Clin. Chim. Acta, 29: 27 (1970).

3. Brown. A. K and Zuelzer, W. W.: Studies on the neonatal development of the glucuronide conjugating system. J. Clin. Invest., 37: 332 (1958).

4. Davies, M. D., and Dickinson, D. B.: Radiochemical assay for UDP-glucose dehydrogenase. Anal. Biochem., 47: 209 (1972).

5. Dutton. G. J.: Glucuronide synthesis in foetal liver and other tissues. Biochem. J., $71: 141$ (1959).

6. Dutton, G. J.: The biosynthesis of glucuronides. In: G. J. Dutton: Glucuronic Acid. Free and Combined, Chemistry. Biochemistry. Pharmacology, and Medicine. p. 185 (Academic Press. New York, 1966).

7. Dutton, G. J.: Control of UDP-glucuronyltransferase activity. Biochem. Pharmacol.. 24: 1835 (1975).

8. Felsher, B. F., Carpio. N. M., Woolley, M. M., and Asch, M. J.: Hepatic bilirubin glucuronidation in neonates with unconjugated hyperbilirubinemia and congenital gastrointestinal obstruction. J. Lab. Clin. Med.. 83: 90 (1974).

9. Fevery, J., Leroy, P., Van de Vijver, M.., and Heirwegh. K. P. M.: Structures of bilirubin conjugates synthesized in vitro from bilirubin and uridine diphosphate glucuronic acid, uridine diphosphate glucose. or uridine diphosphate xylose by preparations from rat liver. Biochem. J., 129: 635 (1972).

10. Fevery, J. R., Van Damme, R., Michiels. R., De Groote, J., and Heirwegh, K. P. $M$.: Bilirubin conjugates in bile of man and rat in the normal state and in liver disease. J. Clin. Invest.. 51: 2482 (1972).

11. Gordon. E. R., Goresky. C. A.. Chang. T., and Perlin, A. S.: The isolation and characterization of bilirubin diglucuronide, the major bilirubin conjugate in dog and human bile. Biochem. J., 155: 477 (1976).

12. Heirwegh, K. P. M., Van de Vijver, M., and Fevery, J.: Assay and properties of digitonin-activated bilirubin uridine diphosphate glucuronyltransferase from rat liver. Biochem. J., 129: 605 (1972).

13. Jansen, P. L. M., Chowdhury. J. R., Fischberg, E. B., and Arias, I. M.: Enzymatic conversion of bilirubin monoglucuronide to diglucuronide by rat liver plasma membranes. J. Biol. Chem., 252: 2710 (1977).

14. Kuenzle. C. C.: Bilirubin conjugates of human bile. Biochem. J., /19: 411 (1970).

15. Lathe, G. H., and Walker, M.: The synthesis of bilirubin glucuronide in animal and human liver. Biochem. J.. 70: 705 (1958).

16. Lowry, O. H., Rosebrough, N. J., Farr, A. L., and Randall, R. J.: Protein measurement with the Folin phenol reagent. J. Biol. Chem., 193: 265 (1951).

17. Streeter, G. L.: Weight, sitting height, head size, foot length, and menstrual age of the human embryo. Contrib. Embryol. (Carnegie Inst.). 9: 143 (1920).

18. Strominger, J. L.. Kalckar, J.. Axelrod, J., and Maxwell, E. S.: Enzymatic oxidation of uridine diphosphate glucose to uridine diphosphate glucuronic acid. J. Amer. Chem. Soc., 76: 6411 (1954).

19. Van Roy. F. P., and Heirwegh. K. P. M.: Determination of bilirubin glucuronide and assay of glucuronyl transferase with bilirubin as acceptor. Biochem. J.. 107: $507(1968)$

20. Wishart. G. J., and Dutton, G. J.: Precocious development in utero of certain UDP-glucuronyltransferase activities in rat fetuses exposed to glucocorticoids. Biochem. Biophys. Res. Commun., 75: 125 (1977).

21. This research was supported in part by Research Grant AM 18088 from the NIH. USPHS, and the Medical Research Programs of the VA.

22. Requests for reprints should be addressed to: B. Felsher. M.D., Hepatology Section. Veterans Administration Hospital, 5901 East 7th Street. Long Beach, CA 90822 (USA).

23. Received for publication August 2, 1977.

24. Accepted for publication October 26. 1977. 